\title{
Tromboembolismo pulmonar con compromiso hemodinámico: Tratamiento con trombólisis farmacológica y fragmentación mecánica. Caso clínico
}

\author{
Mario Fava P, Soledad Loyola Z, Luis Meneses Q . \\ Pulmonary thromboembolism treated \\ with pharmacological thrombolysis \\ and mechanical fragmentation. \\ Report of one case
}

Pulmonary Embolism (PE) has a wide clinical spectrum. It is imperative to detect patients with a high risk to develop right ventricular failure, because this is the main cause of death in patients with massive PE. In this group of patients, invasive therapies to relieve pulmonary obstruction and right ventricle overload should be used as soon as possible. We report a 85 years old male with massive PE treated with pharmacological thrombolysis and mechanical fragmentation with an angioplasty balloon. Pulmonary perfusion improved significantly. Afterwards, systemic anticoagulation was started and an inferior vena cava filter was installed percutaneously. The patient was discharged in good conditions, five days after admission (Rev Méd Chile 2005; 133: 1477-82).

(Key Words: Angioplasty, balloon; Thromboembolism; Thrombolytics therapy)

Recibido el 11 de mayo, 2005. Aceptado el 15 de julio, 2005.

Radiología Intervencional y Terapia Percutánea, Departamento de Radiología, Hospital Clínico Pontificia Universidad Católica de Chile.

$\mathrm{E}^{1}$ tromboembolismo pulmonar (TEP), tiene un amplio espectro de presentación clínica. Es clave identificar aquellos pacientes con TEP masivo, ya que la mortalidad es muy alta de no mediar

$\overline{\text { Correspondencia a: Dr. Mario Fava P. Servicio de Radiología, }}$ Hospital Clínico Pontificia Universidad Católica de Chile. Marcoleta 367, Santiago. Fono: 3543486. Fax: 6336762. E mail: fava@med.puc.cl una adecuada y pronta intervención terapéutica ${ }^{1}$. Presentamos un paciente con TEP masivo, tratado mediante trombólisis farmacológica y fragmentación mecánica.

\section{CASO CĹNICO}

Paciente masculino, 85 años, sin antecedentes mórbidos, salvo resección prostática antigua. El 
paciente presentó traumatismo en tobillo izquierdo 3 semanas previas al ingreso, por lo que permaneció en reposo siete días. Progresivamente, notó aumento de volumen de extremidad inferior izquierda. El día del ingreso, comenzó con dolor retroesternal de moderada intensidad, asociado a lipotimia y disnea. Consultó en el servicio de urgencia, constatándose hipotensión arterial $(70 / 40 \mathrm{mmHg})$, con mala perfusión periférica, frecuencia cardíaca de 60 por minuto, ritmo regular, yugulares ingurgitadas, sin actividad palpable de ventrículo derecho (VD), frecuencia respiratoria de 20 por min y edema de extremidad inferior izquierda. Entre los exámenes de laboratorio destacaba, $\mathrm{PaO}_{2}$ 96,4 $\mathrm{mmHg}$, $\mathrm{PaCO}_{2} 33$ mmHg, pH: 7,39, $\mathrm{HCO}_{3}$ 20,1 $(\mathrm{mmol} / \mathrm{l})$, lactato: $2,6(\mathrm{mmol} / \mathrm{l})$ y diferencia alvéolo-arterial de oxígeno: $29 \mathrm{mmHg}$.

El electrocardiograma mostraba bloqueo completo de rama derecha. Los diagnósticos planteados al ingreso fueron shock cardiogénico por infarto miocárdico de VD versus shock obstructivo por TEP masivo.

El ecocardiograma de superficie demostró marcada dilatación de VD, reflujo tricuspídeo y presión estimada de arteria pulmonar de 65 $\mathrm{mmHg}$, sin comunicación interventricular. El ventrículo izquierdo aparecía normal, con función sistólica conservada.

Se realizó angiografía por tomografía axial computada (angioTAC) de arterias pulmonares, que demostró TEP bilateral, que comprometía arterias pulmonares principales, con dilatación de VD (Figura 1), y reflujo del contraste hacia la vena cava inferior, hallazgos que indicaban disfunción ventricular derecha. La venografía por TAC demostró trombosis venosa profunda ilíaca y femoropoplítea izquierdas. El paciente fue llevado directamente desde scanner al pabellón de angiografía, ingresando en shock refractario a drogas vasoactivas, con saturación de oxígeno de $70 \%$. La angiografía por sustracción digital confirmó el diagnóstico de TEP masivo bilateral (Figura 2). Se realizó fragmentación mecánica de los trombos con balón de angioplastia y un guía angiográfico, obteniendo rápida mejoría en la saturación de oxígeno y los parámetros hemodinámicos.
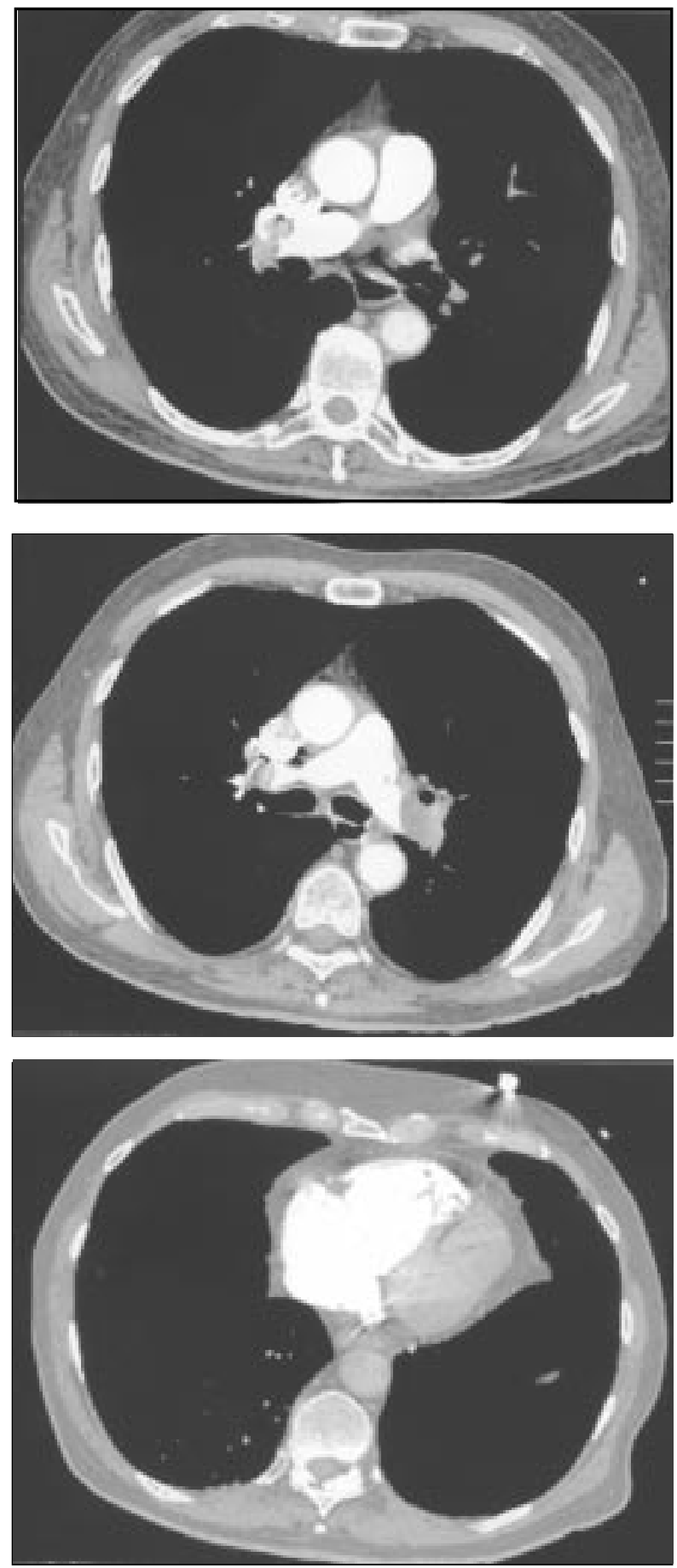

Figura 1. a) y b) Se observan trombos en el interior de ambas arterias pulmonares principales. c) Signos de sobrecarga ventricular derecha: dilatación de ventrículo derecho y desviación a izquierda del tabique interventricular. 


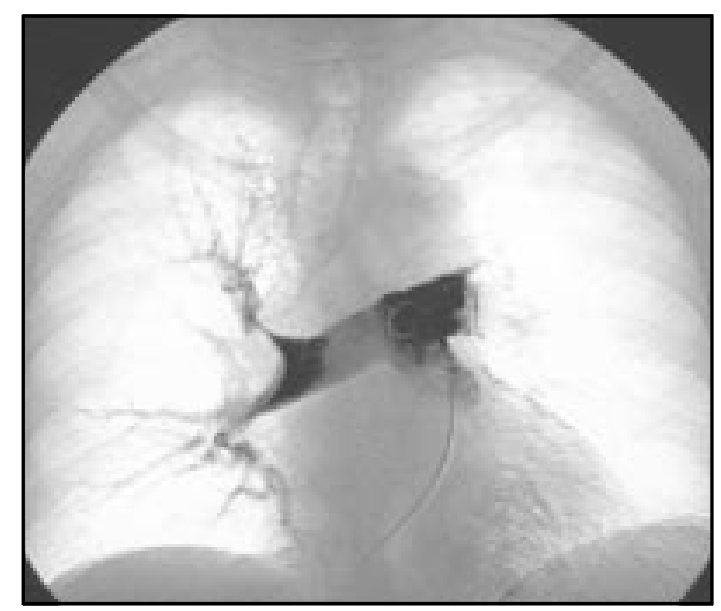

Figura 2a. Angiografía pulmonar inicial, muestra TEP masivo central bilateral.

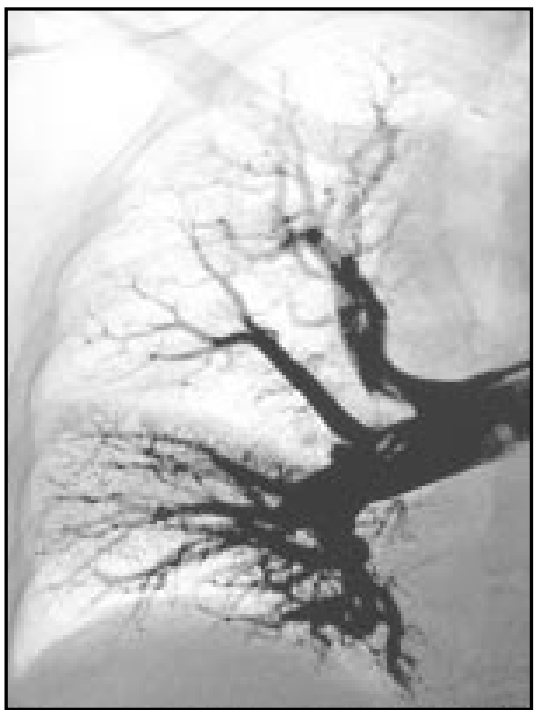

Figura 2b. Control a las $12 \mathrm{~h}$. Significativa mejoría en la perfusión pulmonar.

Además se realizó trombólisis local con activador tisular del plasminógeno (t-PA) $(20 \mathrm{mg})$ y luego infusión de urokinasa a $60.000 \mathrm{U} / \mathrm{h}$ por $12 \mathrm{~h}$. La presión media de arteria pulmonar, medida en forma directa, bajó de 47 a $30 \mathrm{mmHg}$ posterior a la trombólisis.

El paciente presentó gingivorragia y epistaxis leves post trombólisis, que cedieron espontáneamente. En el control final se comprobó significativa mejoría en la perfusión pulmonar. Dada la extensa trombosis venosa profunda en extremidad

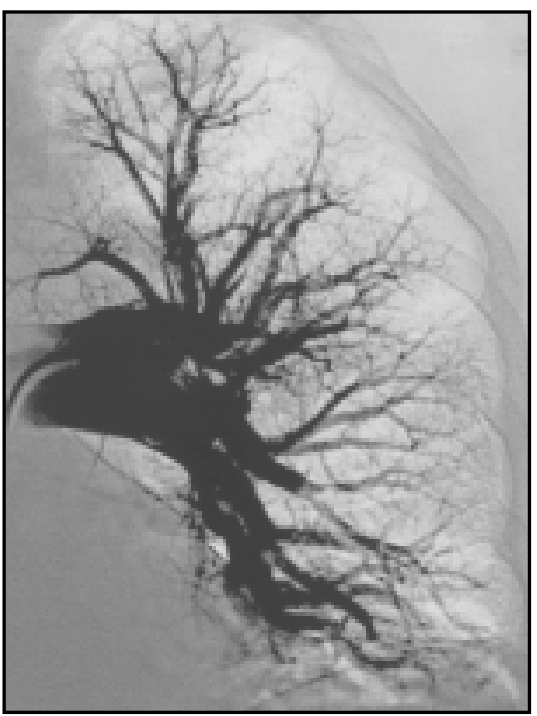

Figura 2c. Angiografía post tratamiento, con reperfusión de arteria pulmonar derecha.

inferior izquierda se instaló, percutáneamente, un filtro de vena cava inferior, sin complicaciones. El paciente inició heparina endovenosa y al segundo día comenzó tratamiento anticoagulante oral (TACO) con acenocumarol.

El paciente fue dado de alta al quinto día de hospitalización, en buenas condiciones generales, permaneciendo en TACO por seis meses. En el control clínico efectuado a 6 meses, se encontraba en buenas condiciones, en capacidad funcional I, sin complicaciones derivadas del TACO. 


\section{DisCUSIÓN}

El cuadro clínico del TEP comprende desde pacientes relativamente asintomáticos a pacientes en shock cardiogénico, de modo que la estratificación de la magnitud del TEP es clave para el adecuado manejo de esta patología; así, aquellos pacientes con TEP de menor magnitud podrían ser tratados con anticoagulación, mientras que aquellos con compromiso hemodinámico requieren de tratamientos más invasivos que reviertan la obstrucción rápidamente ${ }^{1}$.

A pesar de los avances en los métodos diagnósticos y terapéuticos del TEP, en los pacientes con TEP masivo, que produce compromiso hemodinámico, la mortalidad persiste siendo alta $(25 \% \text { a } 65 \%)^{1,2}$. La obstrucción al flujo pulmonar produce elevación de la presión pulmonar, dilatación del ventrículo derecho, que se vuelve hipoquinético y finalmente claudica, y además, disminución del gasto cardíaco, que lleva finalmente a la muerte ${ }^{3}$. EI compromiso pulmonar depende del volumen de los émbolos y de la función pulmonar de base, así, a mayor cantidad de émbolos, mayor es el compromiso hemodinámico y si existe daño cardíaco o pulmonar previo, menor cantidad de émbolos producirán compromiso hemodinámico ${ }^{3}$.

La ecocardiografía es un examen eficiente para diagnosticar la disfunción ventricular derecha. La disfunción ventricular derecha se ha demostrado como un factor de mal pronóstico, con mayor mortalidad, de manera que los pacientes con TEP asociado a sobrecarga ventricular derecha, debieran ser tratados con mayor celeridad ${ }^{4,5}$.

Los tratamientos descritos en TEP con compromiso hemodinámico son la trombólisis farmacológica sistémica o intratrombo, la fragmentación mecánica, la trombectomía mecánica percutánea y la embolectomía quirúrgica ${ }^{6-8}$.

La trombólisis intratrombo permite administrar menor dosis y entregar mayor concentración del trombolítico en el trombo que la trombólisis sistémica. Además, tiene la posibilidad de asociarla a fragmentación de los trombos o trombectomía, lo que aumenta la velocidad y eficacia de la recanalización.

Algunos estudios publicados han demostrado que al menos $50 \%$ de los pacientes con TEP presentan contraindicaciones para el uso de dro- gas trombolíticas ${ }^{9}$, de modo que las restantes técnicas son las opciones terapéuticas en estos pacientes.

La fragmentación mecánica puede efectuarse con elementos disponibles en cualquier pabellón angiográfico, es decir, guías, catéteres diagnósticos y balones de angioplastia. La fragmentación permite desobstruir las arterias pulmonares centrales y dado que la suma del área de sección transversal de las arterias pulmonares periféricas es cuatro veces mayor al área de sección transversal de las arterias pulmonares centrales, basta con fragmentar para reducir significativamente la hipertensión pulmonar y la sobrecarga ventricular derecha ${ }^{10}$. Además, al fragmentar los trombos, aumenta la superficie de contacto con el trombolítico, lo que mejora su eficacia ${ }^{10}$.

La trombectomía mecánica percutánea comprende un grupo de técnicas y dispositivos que permiten micropulverizar y extraer trombos, y que han demostrado mejorar la sobrevida de estos pacientes. Una de estas series publicadas, evidenció una mejoría en 90\% de los pacientes con TEP masivo tratados con trombectomía mecánica percutánea, los que fueron dados de alta en 11 días promedio ${ }^{8}$.

Este paciente ilustra la eficacia del tratamiento del TEP masivo en shock, con trombólisis farmacológica intratrombo y fragmentación mecánica, con recuperación rápida y completa. Es fundamental realizar el procedimiento de la manera más diligente posible, para evitar la claudicación VD. La respuesta terapéutica de la presión de arteria pulmonar en este paciente, que descendió de $45 \pm$ $14 \mathrm{mmHg}$ a $30 \pm 13 \mathrm{mmHg}$, está dentro de los rangos descritos en la literatura. También se describe inmediata mejoría en el intercambio gaseoso, ya durante la realización del procedimiento, como ocurrió en este caso ${ }^{8}$.

Los pacientes con shock de causa desconocida requieren una evaluación adecuada y oportuna. Antiguamente, la cintigrafía y la angiografía pulmonar eran los métodos de elección para el diagnóstico de esta patología ${ }^{11}$, posteriormente algunos trabajos propusieron la ecocardiografía transesofágica, sin embargo, con el advenimiento del angioTAC, este concepto ha cambiado radicalmente. En la actualidad, el algoritmo diagnóstico para los pacientes con sospecha clínica de TEP agudo se inicia con angioTAC, que permite visua- 
lizar tamaño y extensión de los trombos, y entrega signos indirectos de disfunción ventricular derecha, además de otros diagnósticos alternativos que expliquen la sintomatología ${ }^{12,13}$. Una vez certificado el diagnóstico, la recomendación es estratificar el riesgo con una ecocardiografía, para certificar o descartar sobrecarga VD. Actualmente la cintigrafía se propone en el algoritmo de diagnóstico del TEP sólo en caso de contraindicaciones para el uso de contraste yodado ${ }^{14}$.

$\mathrm{Si}$ el compromiso hemodinámico es mayor e impide realizar un angioTAC, el paciente debiera ir idealmente al pabellón de angiografía para confirmar el diagnóstico angiográficamente y recanalizar rápidamente las arterias pulmonares. Una vez diagnosticado el TEP en pacientes con compromiso hemodinámico, el objetivo fundamental es revertir la obstrucción de las arterias pulmonares. Bojan et $\mathrm{al}^{15}$, demostró en un estudio prospectivo, una significativa y rápida disminución de la resistencia y presión de arteria pulmonar, así como importante mejoría en el gasto cardíaco y en la presión sistólica sistémica, inmediatamente des-

\section{REFERENCIAS}

1. Kasper W, Konstantinides S, Geibel A, Olschewski M, HeinRich F, Grosser KD et al. Management strategies and determinants of outcome in acute major pulmonary embolism: results of a multicenter registry. J Am Coll Cardiol 1997; 30: 116571.

2. Goldhaber SZ, De Rosa M, Visan L International cooperative pulmonary embolism registry detects high mortality rate [abstract]. Circulation 1997; 96(suppl): 1591.

3. Wood KE. Major pulmonary embolism: Review of a pathophysiologic approach to the golden hour on hemodynamically significant pulmonary embolism. Chest 2002; 121: 877-905.

4. Ribeiro A, Lindmarker P, Juhin Dannfelt A, Johnson $\mathrm{H}$, JoRFELDS L. Echocardiography Doppler in pulmonary embolism: right ventricular dysfunction as a predictor of mortality rate. Am Heart J 1997; 134: 479-87. pués del uso de terapia trombolítica, hecho que también ocurrió en el paciente presentado en esta revisión. Un hecho interesante, demostrado por estos autores, es que a diferencia de la presión de arteria pulmonar, la presión venosa central no presentó variaciones con el tratamiento trombolítico, por lo que este parámetro puede no ser útil en la evaluación de estos pacientes.

En resumen, las recomendaciones de diagnóstico por imágenes actuales en pacientes con sospecha clínica, sugieren iniciar el estudio con angioTAC de tórax; una vez confirmado el diagnóstico, efectuar ecocardiografía para evaluar compromiso de cavidades derechas y así estratificar el riesgo. Si no presentan compromiso ventricular derecho (bajo riesgo), entonces considerar sólo tratamiento anticoagulante oral; si se evidencia sobrecarga ventricular derecha (alto riesgo), entonces la recomendación es usar terapias percutáneas intervencionales que permiten revertir rápidamente la falla ventricular derecha y así cambiar el curso natural de esta patología ${ }^{14}$ ya que el TEP, así como el infarto miocárdico y el trauma, también tiene su hora dorada»?.

5. Kasper W, Konstantinides S, Geibel A, Tiede N, Krause T, Just H. Prognostic significance of right ventricular afterload stress detected by echocardiography in patients with clinically suspected pulmonary embolism. Heart 1997; 77: 346-9.

6. Fava M, Loyola S, Flores P, Huete I. Mechanical fragmentation and pharmacological thrombolysis in massive pulmonary embolism. JVIR 1997; 8: 261-6.

7. TerRIN M, Goldhaber SZ, ThOMpon B. Selection of patients with acute PE for thrombolytic therapy: Thrombolysis in PE (TIPE) patients survey. The TIPE Investigators. Chest 1989; 95(suppl 5): 279S-281S.

8. Fava M, Loyola S, Huete I. Massive Pulmonary Embolism Treatment with Hydrolyser Thrombectomy Catheter. JVIR 2000; 11: 1159-64.

9. GoLDHABER SZ. Pulmonary embolism. NEngl J Med 1998; 339: 93-104.

10. Singhal S, Henderson R, Horsfield K, Harding K, Cumming G. Morphometry of the human pulmonary arterial tree. Circ Res 1973; 33: 190-7. 
11. PIOPED InveSTIGators. Value of the ventilation/ perfusion scan in acute pulmonary embolism: results of the Prospective Investigation of Puimonary Embolism Diagnosis (PIOPED). JAMA 1990; 263: 2753-9.

12. Eng J, Krishnan JA, Segal JB, Bolger DT, Tamariz LJ, StreifF MB ET al. Accuracy of CT in the Diagnosis of Pulmonary Embolism: A Systematic Literature Review. Am J Roentgenol 2004; 183: 1819-27.

13. Revel MP, Petrover D, Hemigou A, Lefort C, Meyer G, Frija G. Diagnosing Pulmonary Embolism with
Four-Detector Row Helical CT: Prospective Evaluation of 216 Outpatients and Inpatients. Radiology 2005; 234: 265-73.

14. Goldhaber SZ. Pulmonary Embolism. Lancet 2004; 363: 1295-305.

15. Krivec B, Voga G, Zuran I, Skale R, Pareznik R, Podbregar M, Noc M. Diagnosis and Treatment of Shock Due to Massive Pulmonary Embolism. Approach with Transesophageal Echocardiography and intrapulmonary trombolisis. Chest 1997; 112: 1310-16. 